\title{
T RACKING UNMODELLED SIGNALS OF NONLINEAR SYSTEMS VIA ROBUST SLIDING MODE OBSERVER: APPLICATION TO REACTING SYSTEMS
}

\author{
R. Aguilar-López'.2. G. Soto-Cortés', M.I. Nería-González² \& R. Escarela-PéreZ' \\ ${ }^{1}$ Departamento de Energía \\ Universidad Autónoma Metropolitana-Azcapotzalco \\ Av. San Pablo No. 180, Reynosa-Tamaulipas, 02200; Azcapotzalco, México, D.F. MEXICO \\ E-mail.- raguilar@correo.azc.uam.mx \\ Fax.- + (52) 53947378 \\ ${ }^{2}$ Departamento de Biotecnología y Bioingeniería \\ CINVERSTAV-IPN
}

\begin{abstract}
The problem of the on-line estimation of the reaction heat in a continuous stirred tank reactor from temperature measurements is addressed in this paper. The proposed uncertainty observer is based on differential algebraic techniques, such that the algebraic observability condition of the uncertainty from noisy temperature measurements is easily verified and the observer structure is very simple, which lead to feasible implementation. The observer proposed is robust against noisy measurements and sustained disturbances. The good performance of the observer is shown by means of numerical simulations.
\end{abstract}

KEYWORDS: uncertainty observer, noisy measurements, sustained disturbances, robustness

\section{INTRODUCTION}

The Continuous Stirred Tank Reactors (CSTR's) are widely used in the chemical industry, e. g. in polymerization, petrochemical, pharmaceutical, biochemical, etc. Therefore, their importance in the industry is great, nowadays the demanding conditions in security, efficiency, environmental restrictions and so on makes that the process engineers applies more sophisticates techniques in modeling, monitoring and control strategies in order to obtain high performance in the processes.

Generally speaking, the evaluation of reaction heats is a difficult task in chemical processes, due the complexity of the physic-chemical phenomena related. This lead to construct uncertain mathematical models of this process, such that the problem of on-line estimation of reaction heats must to be tackled. Currently, estimation theory is one of the most active research areas to the necessity to obtain on-line estimates of unknown terms related to mathematical models for process identification and control purposes. Schuler and Schmith [1], uses an uncertainty estimator based on calorimetric balances to infer the reaction heats in chemical reactors, Alvarez, et. al. [2] used this estimation methodology coupled with a linearizing controller to regulate temperature in FCC Units, like Aguilar, et. al. [3] whom employ this control scheme too, for the substrate regulation in a continuous bioreactor, but for dynamic estimation of uncertain terms these class of strategies become unstable when the measurements are noisy, because the derivative related with the accumulation terms can not be calculated adequately, which can lead to poor closed-loop performance or instabilities in the process.

Georgakis, et. al. [4] proposed a Kalman filtering technique to estimate kinetics terms in a polymerization reactor, with good results and following this research line Aguilar, et. al. [5] using filtering techniques, design a nonlinear controller based on observer for the regulation of temperature in a CSTR with complex behavior. In this kind of estimation methodologies based on observer Kalman structures, the convergence analysis of the 
observer is difficult because the observer gain is based on an approximation of the covariance matrix related with the estimation error.

\section{MATHEMATICAL BACKGROUND}

In the beginning of the century Ritt [6] introduced the differential algebra with the main idea related to bring the theory of systems of differential equations which are algebraic in the unknowns and their derivatives some of the completeness enjoyed by the theory of systems of algebraic equations. This mathematical approach has recently been shown to be a most effective tool for understanding basic questions such as input-output inversions and realizations [7]-[9]. Now, before to show the estimation methodology proposed, the following definitions must be considered [10], [11]:

Definition 1.- A differential field extension $L / K$ is given by two differential field, $L$ and $K$, such that:

$K \subseteq L$ The derivation of $K$ is the restriction to $K$ of the derivation of $L$.

Definition 2.- Let $u$ be a differential scalar indeterminate and let $k$ be a differential field, with derivation denoted by $d() / d t$.

Definition 3.- A dynamics is a finitely generated differentially algebraic extension $\mathfrak{I} / k<u>$. This means that any element of $\mathfrak{I}$ satisfies a differential algebraic equation with coefficients, which are rational functions over $\mathrm{k}$ in the components of $\mathrm{u}$ and a finite number of their time derivatives.

Definition 4.- Let a subset $\{u, y\}$ of $\mathfrak{I}$ in a dynamics $\mathfrak{I} / k<u>$. An element in $\mathfrak{I}$ is said to be observable with respect to $\{u, y\}$ if it is algebraic over $k<u, y>$. Therefore a state $x$ is said observable if, and only if, it is observable with respect to $\{u, y\}$.

Definition 5.- A dynamics $\mathfrak{I} / \mathrm{k}<\mathrm{u}>$ with output $\mathrm{y}$ is said to be observable if, and only if, any state is so.

\section{PROBLEM STATEMENT}

Consider the following nonlinear dynamic system, related with a mathematical model of a continuous stirred tank reactor [12]:

Mass Balance:

$$
\dot{X}_{o}=\theta\left(X_{o e}-X_{o}\right)-K X_{o}^{2}
$$

Energy Balance:

$$
\dot{X}_{1}=\theta\left(X_{1 e}-X_{1}\right)+X_{2}+\gamma\left(u-X_{1}\right)
$$

Uncertainty dynamics (reaction heat):

$$
\dot{X}_{2}=f\left(X_{1}, X_{2}\right)
$$

System output:

$$
Y=X_{1}
$$

where $X_{0}$ is the reactive concentration, $K$ is the kinetic constant, $X_{1}$ is the reactor temperature, $X_{2}$ is the uncertain term related with the heat generation by chemical reaction, $Y$ is the system output, $u$ is the system input (temperature of the cooling jacket) and $\theta$ and $\gamma$ are the inverse of the residence time and the heat transfer global coefficient, respectively.

We consider the subsystem given by equation (2)-(4). From this, the following differential-algebraic equations can be obtained: 


$$
\begin{aligned}
& X_{1}-Y=0 \\
& \dot{Y}+(\theta+\gamma) Y-\theta X_{1 e}-\gamma u-X_{2}=0
\end{aligned}
$$

Now, It is introduced a new concept called uncertainty algebraically observable:

Definition 6.- An element $X_{u}$ in $\mathfrak{I}$ is said to be uncertainty algebraically observable if $X_{u}$ satisfies a differential algebraic equation with coefficients over $k\langle u, y\rangle$.

From definitions 5 and 6 , along with the differential algebraic equations (5)-(6), the pair uncertaintytemperature $i$. e. $\left\{X_{1}, X_{2}\right\}$ is universally observable in the Diop-Fliess sense [11].

The corresponding Input-Output representation of the system (2)-(3) is given by:

$$
\ddot{Y}+(\theta+\gamma) \dot{Y}=\gamma \dot{u}+f\left(X_{1}, X_{2}\right)
$$

which can be represented in a generalized observability canonical form, using the following change of variables:

$$
\eta_{i}=\frac{d^{i-1} Y}{d t^{i-1}}
$$

to obtain:

$$
\begin{aligned}
& \eta_{1}=\eta_{2} \\
& \eta_{2}=\Phi\left(\eta_{1}, \eta_{2}, u\right) \\
& Y=\eta_{1}+\delta
\end{aligned}
$$

Now, as can be seen from the nature of the system given by equation (9), a standard structure of a Luenberger type observer based with a copy of the system plus measurement error correction is not realizable since the term $\Phi$ is unknown.

The following dynamic system is proposed as an observer of system (9) to obtain filtered estimates of $\eta_{1}$ and $\eta_{2}$ :

$$
\begin{aligned}
& \dot{\hat{\eta}}_{1}=\hat{\eta}_{2}-l \tau^{-1}\left(Y-\hat{\eta}_{1}\right) \\
& \dot{\hat{\eta}}_{2}=-l^{2} \tau^{-1}\left(Y-\hat{\eta}_{1}\right)+l^{2} \tau^{-1} \operatorname{sign}\left(Y-\hat{\eta}_{1}\right)
\end{aligned}
$$

where $\tau_{e}>0$ is an estimation time-constant and

$$
\begin{array}{ll}
\operatorname{sign}\left(Y-\hat{\eta}_{1}\right)=1 \quad \text { if } & \left(Y-\hat{\eta}_{1}\right)>0 \\
\operatorname{sign}\left(Y-\hat{\eta}_{1}\right)=0 \quad \text { if } & \left(Y-\hat{\eta}_{1}\right)=0 \\
\operatorname{sign}\left(Y-\hat{\eta}_{1}\right)=-1 \quad \text { if } & \left(Y-\hat{\eta}_{1}\right)<0
\end{array}
$$

finally from equation (6), the reaction heat is evaluated by the following equation:

$$
\hat{X}_{2}=\hat{\eta}_{2}-\theta\left(X_{1 e}-\hat{\eta}_{1}\right)-\gamma\left(u-\hat{\eta}_{1}\right)
$$


the idea to estimate $\eta_{1}$ and filtering it, is that this variable is directly the reactor temperature (system output) and in accord with the equation (12) if the temperature measurements are noisy, the noise would be transmitted to the estimation of the reaction heat, which can lead to poor performance, In this work, it is supposed that the reactor temperature $X_{1} \geq 0$ is bounded for all $t>0$. Consequently, the concentrations inside the reactor are bounded input to bounded output state. It is assumed too, that the uncertain term remains bounded. The restraint of the heat of reaction (uncertain term) is common for a wide class of chemical reactions and is consequence of characteristics of the mathematical modeling commonly employed; chemical reactions are usually Lipschitz with respect to temperature. It is not hard to see that global Lipschitz of $\Delta H_{f} R\left(y_{f} \cdot T_{r}\right)$ property is found if the functionality $R\left(y_{f}, T_{r}\right)$ with respect to temperature is of Arrhenius type.

Defining the estimation errors as:

$$
\begin{aligned}
& e_{1}=\eta_{1}-\hat{\eta}_{1} \\
& e_{2}=\frac{\eta_{2}-\hat{\eta}_{2}}{l}
\end{aligned}
$$

Considering the above equations (13) and (14), the dynamic of the estimation error is defined as:

$$
\dot{E}=l A E+\Omega\left(\eta_{1}, \eta_{2}\right)
$$

Where:

$$
\begin{aligned}
& E=\left[\begin{array}{l}
e_{1} \\
e_{2}
\end{array}\right] \\
& A=\left[\begin{array}{ll}
\tau^{-1} & 1 \\
\tau^{-1} & 0
\end{array}\right] \\
& \Omega=\left[\begin{array}{c}
\delta \\
\frac{\Phi}{m}+m \tau^{-1} \operatorname{sign}\left(e_{1}+\delta\right)+\delta
\end{array}\right]
\end{aligned}
$$

In principle, the presence of the term $\operatorname{sign}\left(e_{1}+\delta\right)$ deserves a stochastic study. Useful insight can be obtained using the following simplifying approximations [13]

A1.- the additive noise $\delta$ is a deterministic signal of bounded spectrum, i.e., $0 \leq \omega<\omega_{-}$or $\omega>\omega_{+} \Rightarrow F_{\delta}(\omega)$ $=0$, where $F_{\delta}(\omega)$ is the Fourier transform of $\delta$.

A2.- The measurement noise is bounded by some constant $\delta_{0}$ (i.e., $\delta \in\left[-\delta_{0}, \delta_{0}\right]$ ).

Considering the average closed-loop error $E_{a}$ of the system, its dynamics can be approximated by the following equation:

$$
\begin{aligned}
& \dot{e_{1 a}}=-m \tau^{-1} e_{1 a}+e_{2 a} \\
& \dot{e_{2 a}}=-m \tau^{-1} e_{1 a}+m \tau^{-1} \text { average }\left(\operatorname{sign}\left(e_{1 a}+\delta\right)\right)+\Phi_{a}
\end{aligned}
$$


where average ( $\left.\operatorname{sign}\left(e_{1 a}+\delta\right)\right)$ is computed over short time periods during which $E_{a}$ is treated as a constant and $\Phi_{\mathrm{a}}$ is defined as average $[\Phi]$. Considering that $\delta$ is white noise, then Average $\left(\operatorname{sign}\left(e_{1 a}+\delta\right)\right)$ $=$ Expectation $\left(\operatorname{sign}\left(e_{1 a}+\delta\right)\right)$, which lead to the following expression:

$$
\text { Expectation }\left(\operatorname{sgn}\left(e_{1 a}+\delta\right)\right)=\int_{-\infty}^{\infty} \operatorname{sign}\left(e_{1 a}+\delta\right) P(\delta) d \delta=2 \int_{0}^{e_{1}} P(\delta) d \delta
$$

where the last equality assumes that the probability density function $P(\delta)$ is symmetric. Thus, the average value is an odd continuous function of $e_{1}$. Considering Assumption A2, the following expression is obtained:

$$
\text { Expectation }\left(\operatorname{sign}\left(\mathrm{e}_{1 \mathrm{a}}+\delta\right)\right)=\frac{\mathrm{e}_{1 \mathrm{a}}}{\delta_{0}}
$$

In this way, the average equations for the estimation errors of the observer are given by:

$$
\begin{aligned}
& \dot{e_{1 a}}=-l \tau^{-1} e_{1 a}+e_{2 a} \\
& \dot{e_{2 a}}=\left(-l \tau^{-1}+\frac{l \tau^{-1}}{\delta_{0}}\right) e_{1 a}+\frac{\Phi_{a}}{l}
\end{aligned}
$$

or, in vector notation,

$$
\dot{E}_{a}=l A E_{a}+\Lambda\left(\eta_{1}, \eta_{2}\right)
$$

where:

$$
\begin{aligned}
& E_{a}=\left[\begin{array}{l}
e_{1 a} \\
e_{2 a}
\end{array}\right] \\
& A=\left[\begin{array}{cc}
-\tau^{-1} & 1 \\
-\tau^{-1}+\frac{-\tau^{-1}}{\delta_{0}} & 0
\end{array}\right] \\
& \Lambda=\left[\begin{array}{c}
0 \\
\frac{\Phi_{a}}{l}
\end{array}\right]
\end{aligned}
$$

Considering the following assumptions:

A1.- $\Omega$ is bounded, i. e. $\|\Omega\| \leq \Gamma$

A2.- There exist two positive constants $j>0$ and $\lambda>0$, such that:

$$
\left\|\exp (l A t) E_{a}\right\| \leq j \exp (-l \lambda t)\left\|E_{a}\right\|
$$

Now, solving the equation (15), the next expression is obtained:

$$
E_{a}=\exp (l A t) E_{a 0}+\int_{0}^{t} \exp \{l A(t-s)\} \Omega d s
$$

Considering the assumptions A1, A2 and taking norms for both sides of the eq. (16), the following equation is generated: 


$$
\left\|E_{a}\right\| \leq j \exp (-l \lambda t)\left[\left\|E_{a 0}\right\|-\frac{j \Gamma}{l^{2} \lambda}\right]+\frac{j \Gamma}{l^{2} \lambda}
$$

in the limit, when $t \rightarrow \infty$ :

$$
\left\|E_{a}\right\| \leq \frac{j \Gamma}{l^{2} \lambda}
$$

The above inequality implies that the estimation error, in average, can be as small as is desired, if the observer gain $/$ is chosen large enough.

\section{NUMERICAL EXPERIMENTS}

In this section, numerical simulations were carried out in order to show the performance of the proposed observer. The reaction heat generated in a continuous stirred tank reactor is estimated via temperature measurements, which are corrupted with a white noise of $\pm 2 \mathrm{~K}$ around the current temperature value. The observer filters adequately the noisy temperature measurements as can be observed in the figure 1 ,

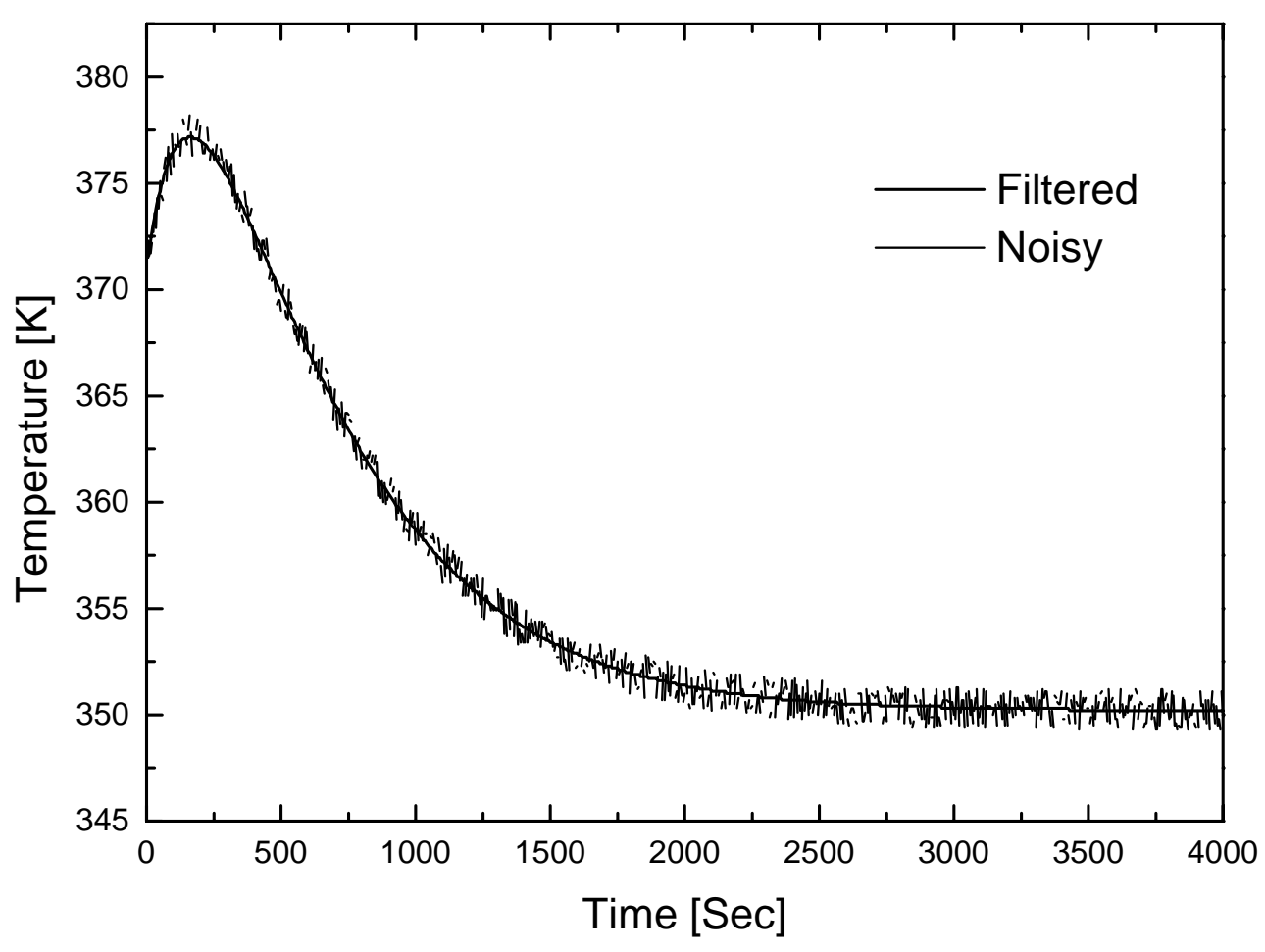

Figure 1. Filtering process of the reactor temperature measurements. 
which are used in the estimation of the reaction heat although the differential algebraic corresponding structure. The observer, is able to infer the reaction heat with a good performance as is shown if the figure 2 .

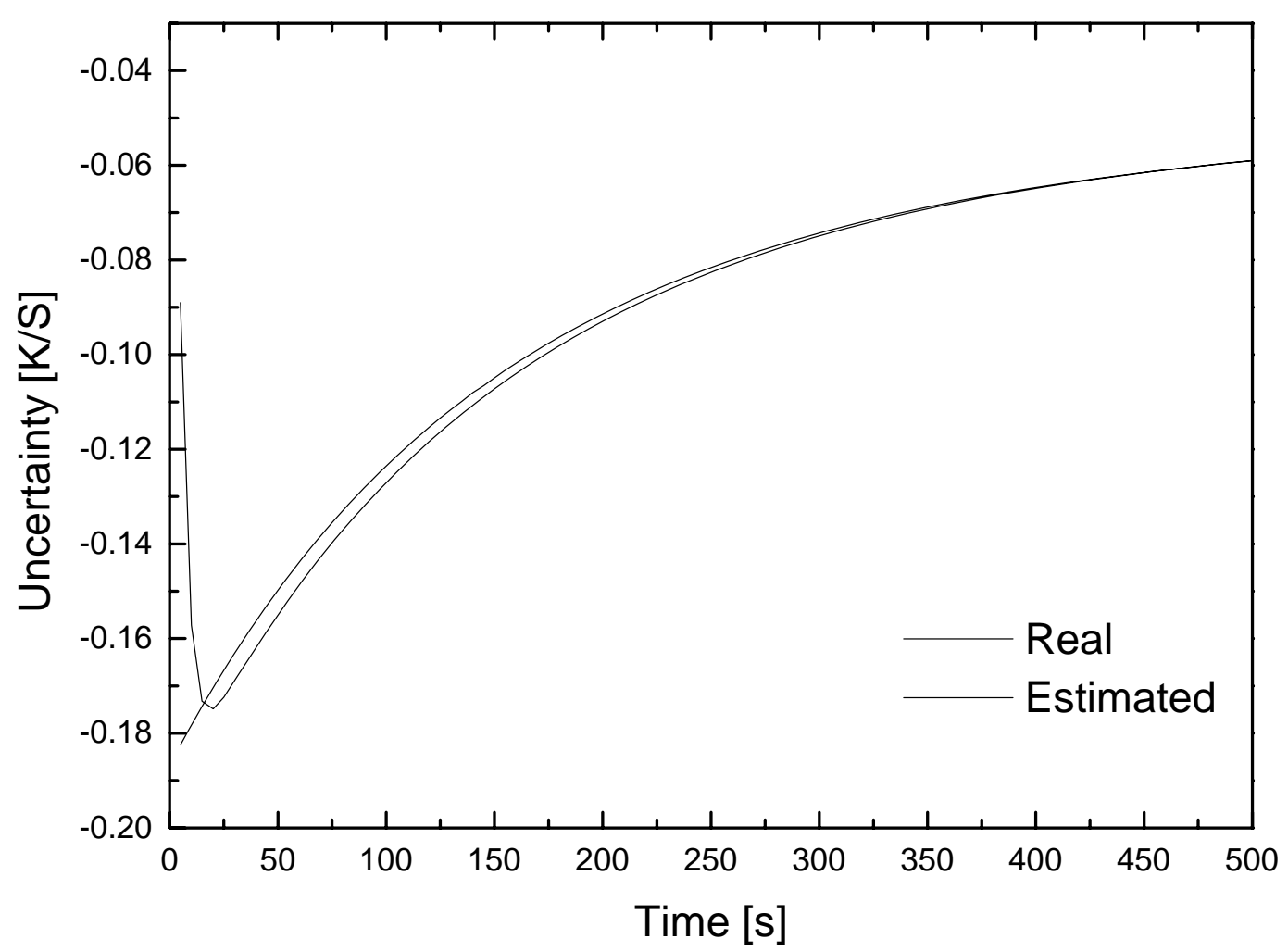

Figure 2. On-line estimation of the reaction heat.

Additionally to the noisy temperature measurements, a sustained disturbance in the reactor temperature inlet $X_{1 e}=X_{1 e o}+4 \operatorname{Sin}(\Pi t)$ is now introduced to the system and the observer proposed is able to estimate the corresponding terms. As can be seen in Figure 3 corresponding to noisy reactor temperature measurement and the related temperature filtered. Figure 4 shown the performance of the observer to infer the reaction heat in the chemical reactor For Figures 1 to 4 the value of the observer parameter gain $I$ is $I=0.01$ and the parameter $\tau=1.0$ 


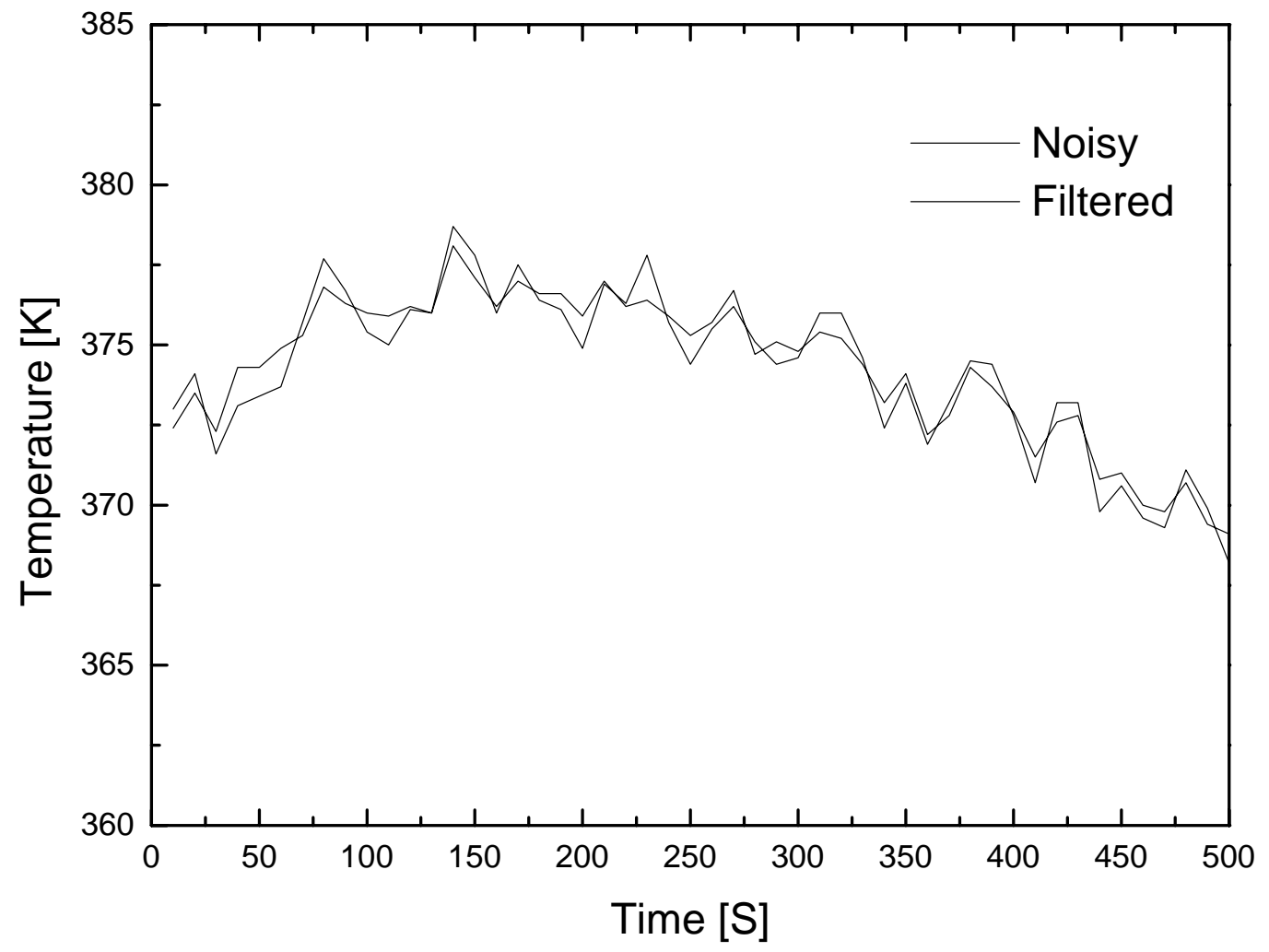

Figure 3. Filtering process of the reactor temperature measurements considering sustained disturbances. 


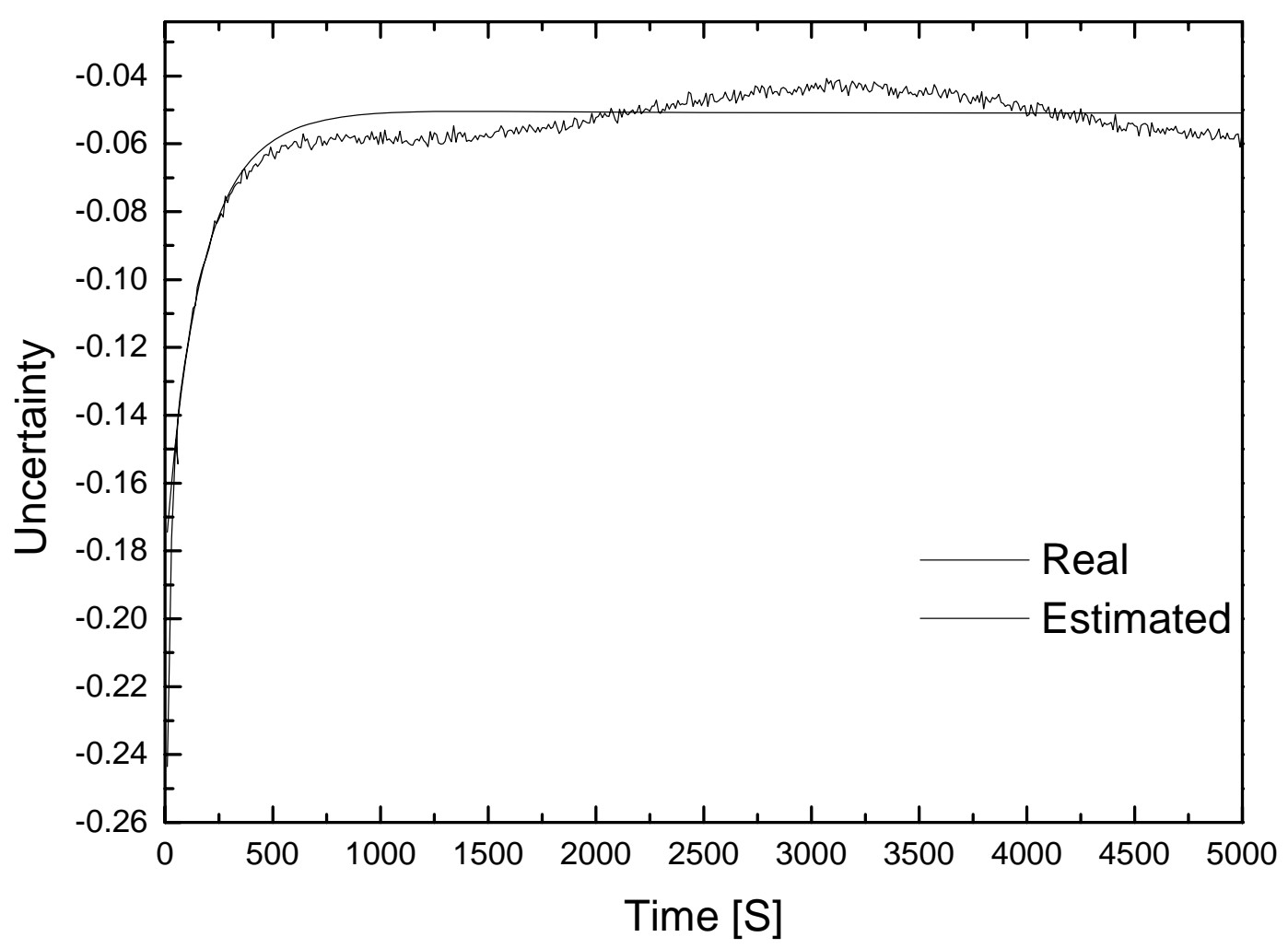

Figure 4. On-line estimation of the reaction heat considering sustained disturbances .

\section{CONCLUDING REMARKS}

A sliding-mode observer to infer reaction heats in a continuous stirred tank reactor via temperature measurements is designed using differential algebraic tools. The concept of uncertainty algebraic observability condition was introduced to estimate the uncertain term from the output selected and is easily obtained from this approach, besides the implementation of the observer is very simple with the transformation proposed. The performance of the observer developed is satisfactory in spite of noisy temperature measurements and sustained disturbances under the high gain condition. 


\section{REFERENCES}

[1] Schuler, H. and Schmidt, C. Calorimetric-state estimator for chemical reactors diagnosis and control: review of methods and applications. Chem. Eng. Sci. (1992), 47, 899-915.

[2] Alvarez-Ramirez, J., Aguilar, R. and López-Isunza. F. Robust regulation of temperature in reactor-regenerator fluid catalytic cracking units. Industrial Engineering Chemistry Research. (1996), 35, 1652-1659.

[3] Aguilar, R., Alvarez Jo., González, J. and Barrón M. A. A strategy to regulate continuous fermentation process with unknown reaction rates. Journal of Chemical Technology and Biotechnology. (1996), 37, 4, 357-361.

[4] Elicabe, G. E., Ozdeger, E. and Georgakis, C. On line estimation of reaction rate in a polymerization process. Ind. Eng. Chem. Res. (1995), 34, 1219.

[5] Aguilar, R., González, J., Alvarez J. and Barrón, M. A. Temperature regulation of a class of continuous chemical reactor based on a nonlinear Luenberger-like observer. Journal of Chemical Technology and Biotechnology. (1997), 70, 3, 209-216.

[6] Ritt,J. F. Differential equations from the algebraic standpoint. Amer. Math Soc. New York. 1932.

[7] Fliess, M. Hasler, M. Questioning the classical state-space description via circuit example. Mathematical Theory of networks and systems. Boston, MA, USA. Birkhauser. 1990.

[8] Hasler, M. Neirynck, J. Nonlinear circuits. Boston, MA, USA. Artech House. 1986.

[9] Freedman, M. I., Willems, J. C. Smooth representation of systems with differentiated inputs. IEEE Transc. Aut. Contr. (1978), 23, 16-21.

[10] Fliess, M. Generalized controller canonical forms for linear and nonlinear dynamics. IEEE Transc. Aut. Contr. (1990), 35, 9, 994-1001.

[11] Martínez-Guerra, R. and León-Morales, J. de. Nonlinear estimators: A differential algebraic approach. Appl. Math. Lett. (19969, 9, 4, 21-25.

[12] Smith, C. A. and Corripio, A. Principles and Practice of automatic process control. John Wiley \& Sons. 1980.

[13] Slotine, J., Hedrick, J. and Misawa, E. On sliding observers for non-linear systems. J. Dyn. Meas. \& Ctrl. (1987), 109, 245-251. 
Authors Biography

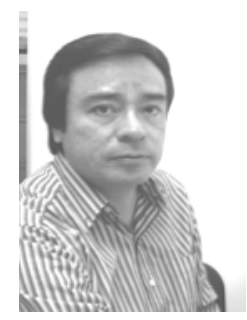

Ricardo Aguilar-López

Born in Mexico City in 1964. He got their B Sc, M Sc and Ph D degrees from Universidad Autónoma Metropolitana in 1989, 1993 and 1998, respectively, all of them in chemical engineering. He was a Postdoctoral Fellow at Instituto Mexicano del Petróleo (2000), besides he ears a Ph D in automatic control from CINVESTAV-IPN (2003). He works in robust observer design for nonlinear systems and process control. He is author and co-author of 67 papers published in international journals. Currently he is research in the Departamento de Biotecnología y Bioingeniería del Centro de Investigación y Estudios Avanzados del IPN and member of the Sistema Nacional de Investigadores since 1998.

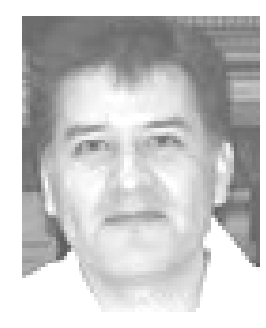

Gabriel Soto-Cortés

Born in México City in 1970, He got the B Sc in physical engineering from Universidad Autónoma Metropolitana (1994), the M Sc and Ph D degrees were obtained from Instituto de Ingeniería, UNAM (1997 y 2000) both two on Hydraulic science. He is author or co-author of 17 papers Publisher in international and national journals. Currently he is professor at Universidad Autónoma Metropolitana, campus Azcapotzalco. He is with the Sistema Nacional de Investigadores since 2001. 


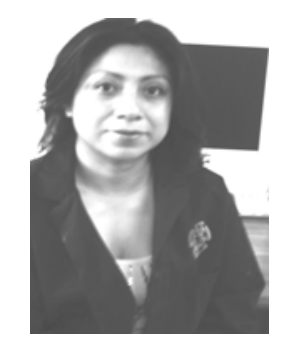

María Isabel Neria-González

Born in México City in 1971, she is Biochemical engineering from Universidad Autónoma Metropolitana (1999), she ears her M sc and Ph D degrees in microbiology science from Escuela Nacional de Ciencias Biológicas del IPN (2002 and 2006, respectively). She is author of 3 published papers in indexed journals.

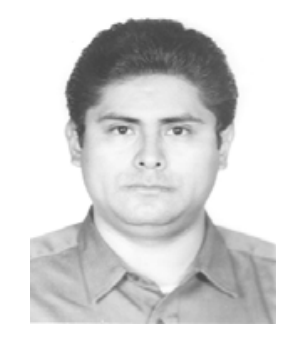

\section{Rafael Escarela-Perez}

Born in México City in 1969. He is Electric engineer from Universidad Autónoma Metropolitana (1992) and he ears his Ph D from Imperial College, London (1996). Currently, Dr. Escarela-Perez is Professor at Departamento de Energía, Universidad Autonoma Metropolitana-Azcapotzalco, Mexico. His research lines are related with numerical modeling of distributed systems. 